\title{
Euler Characteristic of Graphs and Networks
}

\author{
M. EAWNICZAK ${ }^{a}$, P. KURASOV ${ }^{b}$, S. BAUCH ${ }^{a}$, \\ M. BIAŁOUS ${ }^{a}$ AND L. SIRKO ${ }^{a}$ \\ ${ }^{a}$ Institute of Physics, Polish Academy of Sciences, \\ Aleja Lotnikow 32/46, PL-02668 Warsaw, Poland \\ ${ }^{b}$ Department of Mathematics, Stockholm University, S-106 91 Stockholm, Sweden \\ Doi: $10.12693 /$ APhysPolA.139.323 \\ *e-mail: sirko@ifpan.edu.pl
}

\begin{abstract}
The Euler characteristic $\chi=|V|-|E|$ is an important topological characteristic of graphs and networks. Here, $|V|$ and $|E|$ denote the number of vertices and edges of a graph or a network. It has been shown in [Phys. Rev. E 101, 052320 (2020)] that the Euler characteristic can be determined from a finite sequence of the lowest eigenenergies $\lambda_{1}, \ldots, \lambda_{N}$ of a simple quantum graph. We will test this finding numerically, using chaotic graphs with $|V|=8$ vertices. We will consider complete (fully connected) and incomplete realizations of 8-vertex graphs. The properties of the Euler characteristic will also be tested experimentally using the sequence of the lowest resonances of the 5-vertex microwave network. We will show that the Euler characteristic $\chi$ can be used to reveal whether the graph is planar or not.
\end{abstract}

topics: quantum graphs, microwave networks, Euler characteristic

\section{Introduction}

The foundation of graph theory and topology was laid in 1736 by Leonhard Euler [1] who considered the problem of seven bridges of Königsberg. Two hundred years later the idea of graphs was used by Linus Pauling [2] in order to describe quantum particles moving in physical networks. Thereafter, the theory of quantum graphs was extensively developed $[3-7]$.

In this article, we will consider a model of a metric graph $\Gamma=(V, E)$ which is formed by the edges $e \in E$ connected at the vertices $v \in V$. Each edge is an interval on the real line $\mathbb{R}$ having the length $l_{e}$. We will consider the Laplace operator $L(\Gamma)=-\frac{\mathrm{d}^{2}}{\mathrm{~d} x^{2}}[6]$ acting in the Hilbert space of square integrable functions on $\Gamma$. We assume the standard (Neumann or Kirchhoff) vertex conditions imposing continuity of the function and vanishing of the sum of outgoing derivatives at every vertex $v$. Quantum graphs are used to simulate, for example, quantum wires [8], mesoscopic quantum systems $[9,10]$, and optical waveguides [11].

In this article, we test numerically and experimentally the recently published breakthrough results on the Euler characteristic [12], using chaotic graphs with $|V|=8$ vertices. Complete (fully connected) and incomplete realizations of the 8-vertex graphs will be considered. We will show that the Euler characteristic $\chi$ is very sensitive to the internal structure of the graphs. The properties of the Euler characteristic will also be tested experimentally using the sequence of the lowest resonances of a 5 -vertex microwave network.
Quantum graphs can be modeled experimentally by microwave networks [13-18]. It is possible because both systems are described by the same equations. The one-dimensional Schrödinger equation describing quantum graphs is formally equivalent to the telegrapher's equation for microwave networks $[13,16]$. Microwave networks allow for the experimental simulation of systems corresponding to all three classical ensembles in the randommatrix theory (RMT): the systems with $T$ invariance belonging to the Gaussian orthogonal ensemble (GOE) [13-15, 17, 19] and the Gaussian symplectic ensemble (GSE) [20, 21], and the systems without $T$ invariance belonging to the Gaussian unitary ensemble (GUE) [13, 18, 22-25].

The universality of microwave networks has significantly extended research with systems such as flat microwave cavities [26-38] and experiments with the Rydberg atoms strongly driven by microwave fields [39-51] that are successfully used in experimental modeling of complex quantum systems.

The Euler characteristic of a metric graph $\Gamma=(V, E)$ :

$$
\chi=|V|-|E|
$$

determines the number $\beta$ of independent cycles in a graph

$$
\beta=|E|-|V|+1 \equiv 1-\chi .
$$

The number $\beta$ measures how different a graph is from a tree graph and is equal to the number of edges that have to be removed to turn the graph into a tree. 
The total length of a graph

$$
\mathcal{L}=\sum_{e \in E} l_{e}
$$

determines the asymptotics of the graph's eigenvalues $\lambda_{n}$ via Weyl's formula

$$
\lambda_{n}=\left(\frac{\pi}{\mathcal{L}}\right)^{2} n^{2}+\mathcal{O}(n)
$$

where $\mathcal{O}(n)$ is a function which in the limit $n \rightarrow+\infty$ is bounded by a constant.

From the mathematical point of view, the determination of both characteristics requires the knowledge of the whole sequence of eigenvalues [52, 53]. It was proven that the whole spectrum allows one to reconstruct the metric graph, provided that the edge lengths are rationally independent [54-56]. This also means an affirmative answer to a question asked by Mark Kac [57], rephrased in the case of quantum graphs as "Can one hear the shape of a graph?" [15].

In the real world experiments, the whole spectrum of an open system is usually not determinable. In the case of microwave networks, measured at room temperature, the openness of the systems and the existence of intrinsic absorption cause that one may count on measuring up to a hundred of the lowest eigenfrequencies. Therefore, it is important to find out whether the Euler characteristic $\chi$ can be reconstructed directly from the spectrum of the lowest eigenvalues without determining the exact shape of the graph. Since the Euler characteristic $\chi$ is an integer number (often negative), then to determine it precisely it is enough to know its value with an error $\epsilon<1 / 2$. Knowing that only a limited number of eigenvalues can be measured in the experiment, we will use this criterion in our study as a new fast converging formula [12] for the Euler characteristic. Namely,

$$
\chi=\left.\lim _{K \rightarrow \infty} X(t)_{K}\right|_{t \geq t_{0}},
$$

where

$$
X_{K}(t):=2+\sum_{\substack{n=1 ; \\ k_{n} \neq 0}}^{K} \frac{8 \pi^{2} \sin \left(k_{n} / t\right)}{\left(k_{n} / t\right)\left[(2 \pi)^{2}-\left(k_{n} / t\right)^{2}\right]} .
$$

Here, $k_{n}$ are the square roots of the eigenenergies $\lambda_{n}$ and $t_{0}=1 /\left(2 l_{\min }\right)$ with $l_{\text {min }}$ denoting the length of the shortest edge of a simple graph.

In [12], it was additionally shown that in order to evaluate the Euler characteristic $\chi$ using (6), with an error $\epsilon$, it is enough to take the first $K$ eigenvalues. Those eigenvalues can be evaluated using

$$
K \simeq|V|-1+2 \mathcal{L} t_{0}\left[1-\exp \left(\frac{-\pi \epsilon}{\mathcal{L} t_{0}}\right)\right]^{-1 / 2}
$$

The details of the proof are given in [12].

\section{Numerical and experimental results}

The Euler characteristic formula (6) was tested numerically and experimentally using non-planar 8-vertex quantum graphs and planar microwave

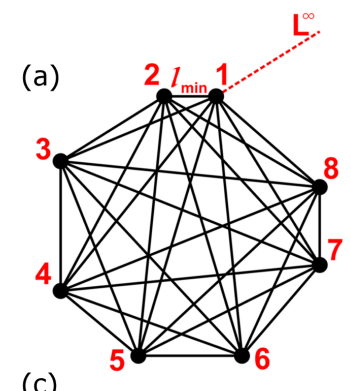

(c)
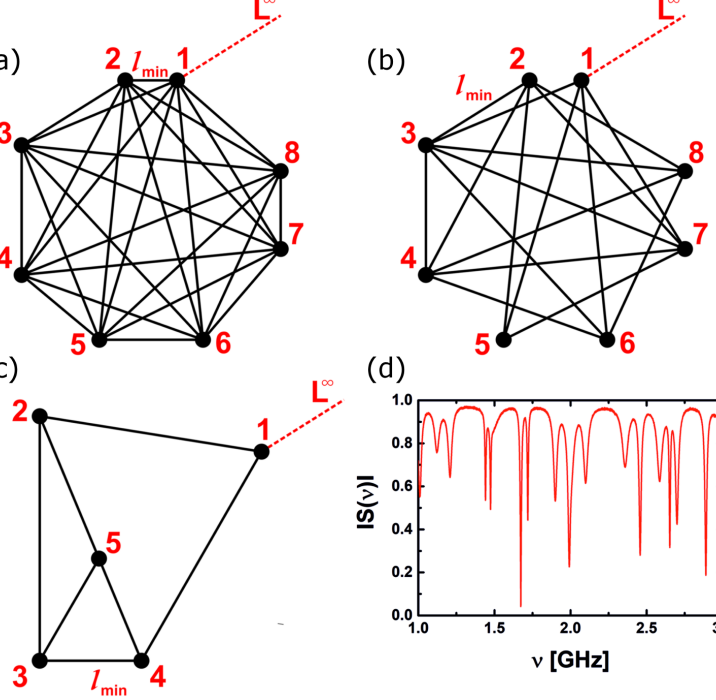

(d)

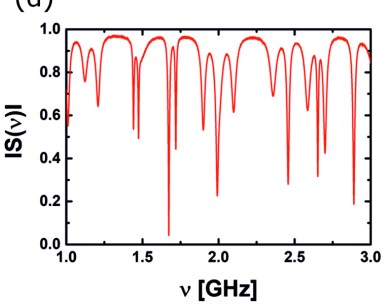

Fig. 1. Parts (a) and (b) present the schemes of two non-planar quantum graphs with $|V|=8$ vertices possessing $|E|=28$ edges and $|E|=18$ edges, respectively. Part (c) shows the schemes of a microwave network planar with $|V|=5$ vertices and $|E|=7$ edges. The microwave network was connected to a vector network analyzer Agilent E8364B with a flexible microwave cable which is equivalent to attaching an infinite lead to a quantum graph. In part (d), we show the examples of the moduli of the scattering matrix $|S(\nu)|$ of the microwave network measured in the frequency range $\nu=1-3 \mathrm{GHz}$.

networks for which the counting function of the number of resonances satisfies Weyl's law [19].

In Fig. 1a and b, we present the schemes of two non-planar quantum graphs with $|V|=8$ vertices possessing $|E|=28$ edges and $|E|=18$ edges with the total lengths $\mathcal{L}=3.916 \mathrm{~m}$ and $\mathcal{L}=2.412 \mathrm{~m}$, respectively.

Figure 1c shows the scheme of the microwave network with $|V|=5$ vertices and $|E|=7$ edges. The total optical length of the network is $\mathcal{L}=1.494 \pm 0.006 \mathrm{~m}$ and the optical length of the shortest edge is $l_{\min }=0.155 \pm 0.001 \mathrm{~m}$. The optical lengths $l_{i}^{\text {opt }}$ of the edges of the network are connected with their physical lengths $l_{i}^{\mathrm{ph}}$ through the relation $l_{i}^{\text {opt }}=\sqrt{\varepsilon} l_{i}^{p h}$, where $\varepsilon=2.06$ is the dielectric constant of Teflon used for the construction of the SMA microwave cables.

The quantum graphs are a closed dissipationless system for which, according to the definition of the Euler characteristic, (6), $\chi=|V|-|E|=-20$ and $\chi=-10$, respectively. The lack of dissipation is a standard assumption considered in the mathematical analysis of graphs.

In Fig. 2 we show the approximation function for the Euler characteristic $X_{K}(t)$ calculated using the first $K=563$ (green full line) and $K=253$ (red full line) resonances of the quantum graphs with $E=28$ and $E=18$ edges, respectively. 


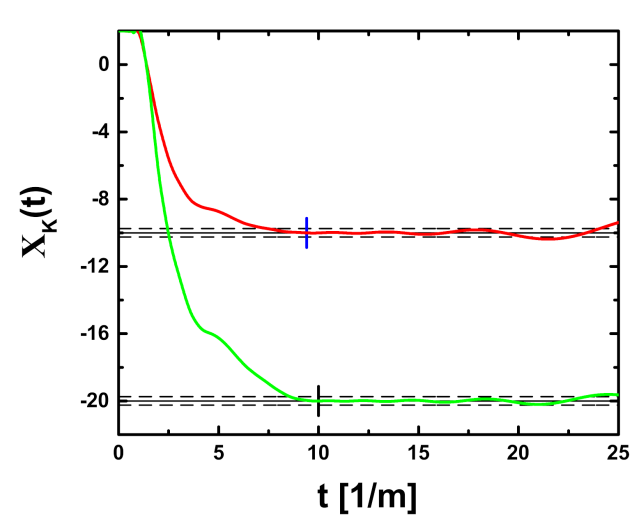

Fig. 2. The approximation function for the $\mathrm{Eu}-$ ler characteristic $X_{K}(t)$ calculated for the 8-vertex fully connected quantum graph with $|E|=28$ edges (full green line). The green curve shows the function $X_{K}(t)$ calculated with (6) for the first $K=563$ resonances. The red full curve shows the Euler characteristic $X_{K}(t)$ calculated for the 8-vertex quantum graph with $|E|=18$ edges using the first $K=253$ resonances. The black and blue vertical marks show the values of $t_{0}=1 /\left(2 l_{\text {min }}\right)=10 \mathrm{~m}^{-1}$ and $t_{0}=1 /\left(2 l_{\min }\right)=9.4 \mathrm{~m}^{-1}$, which were used for the evaluation of the required number of resonances $K=563$ and 253, respectively (see (7)). The black full line shows the expected value of the Euler characteristic $\chi=-20$ and $\chi=-10$, respectively. The black broken lines show the limits of the expected errors $\chi \pm 1 / 4$.

The values $K=563$ and $K=253$ were estimated with (7) taking into account the size of the networks $\mathcal{L} t_{0}=39.16$ and $\mathcal{L} t_{0}=22.67$, respectively, and assuming that $\epsilon=1 / 4$. Our calculations show that the Euler characteristic $\chi$ is a very sensitive indicator of the internal structure of the graphs, easily differentiating graphs with $E=28$ and $E=18$ edges.

We applied (6) to reconstruct the Euler characteristic of the quantum graphs, shown in Fig. 1a and $\mathrm{b}$, using numerically calculated spectra. The obtained correct results are $\chi=-20$ and $\chi=-10$, respectively. We also applied (6) to reconstruct the Euler characteristic of the microwave network in Fig. 1c. The obtained result, i.e., $\chi=-2$, is in agreement with the theoretical one. This system is open but it is characterized by small dissipation [22].

The resonances $\nu_{1}, \ldots, \nu_{N}$ of the microwave network required for the evaluation of the Euler characteristic were determined from the one-port measurements of the scattering matrix $S(\nu)$ of the network using the vector network analyzer (VNA) Agilent E8364B. In this case, the real part of the wave number $k_{n}$ is directly related to the position $\nu_{n}$ of the resonance $\operatorname{Re}\left[k_{n}\right]=\frac{2 \pi}{c} \nu_{n}$. The VNA was connected to the microwave network using the HP 85133-616 microwave flexible cable. From the theoretical point of view, such a situation is equivalent to attaching an infinite lead to a quantum graph [19].

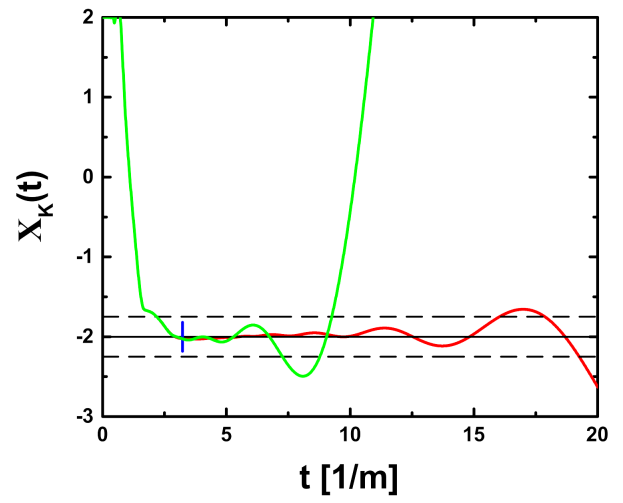

Fig. 3. The approximation function for the Euler characteristic $X_{K}(t)$ calculated for the microwave network with $|V|=5$ vertices and $|E|=7$ edges. The full green and red curves show the Euler characteristic calculated using (7) for the first $K=29$ and $K=100$ resonances, respectively. Estimating the value $K=29$ there was assumed $\epsilon=1 / 4$ and the optical size of the network as $\mathcal{L} t_{0}=4.82 \pm 0.05$. The blue vertical mark shows the value of $t_{0}=1 /\left(2 l_{\min }\right) \simeq 3.23 \pm 0.02 \mathrm{~m}^{-1}$ which was used for the evaluation of the required number of resonances $K=29$ (see (7)). The black full line shows the expected value of the Euler characteristic $\chi=-2$. The black broken lines show the limits of the expected errors $\chi \pm 1 / 4$.

The approximation function for the Euler characteristic $X_{K}(t)(6)$ is shown in Fig. 3. It is evaluated using the first $K=29$ (green full line) and $K=100$ (red full line) experimentally measured resonances of the system. The value $K=29$ was estimated with (7) assuming that $\epsilon=1 / 4$ and taking into account the optical size of the network $\mathcal{L} t_{0}=4.82 \pm 0.05$. In Fig. $1 \mathrm{~d}$, we show the modulus of the scattering matrix $|S(\nu)|$ of the microwave network with $|V|=5$ measured in the frequency range $\nu=1-3 \mathrm{GHz}$. Figure 3 demonstrates that the first $K=29$ resonances (green full line) are enough to identify a plateau close to the expected value $\chi=-2$. The Euler characteristic calculated for the larger number of resonances, i.e., $K=100$ (full red line), displays a much longer plateau along the expected value $\chi=-2$. This also shows that $K=100$ is an excessive number of resonances in relation to what is needed for the practical evaluation of the Euler characteristic.

Importantly, using (6) and (2) one can determine whether a system is planar. In the case of the microwave network, the number of cycles yielded from (2) is $\beta=1-\chi=3$. According to Kuratowski's theorem [58], every non-planar graph should contain $K_{5}$ (the complete graph on 5 vertices) or $K_{3,3}$ (the complete bipartite graph on 3 and 3 vertices) as subgraphs. These graphs have 6 and 4 cycles, respectively. Therefore, we learn that the microwave network considered in this article, even without seeing it, simulates a planar quantum graph. 


\section{Conclusions}

We showed that the Euler characteristic $\chi$ an important topological characteristic of graphs and networks - can be effectively determined by a finite number of lowest eigenvalues. On the examples of 8-vertex quantum graphs, we demonstrated that $\chi$ is very sensitive to the internal structure of the graphs. The properties of the Euler characteristic were also tested experimentally using the sequence of the lowest resonances of the 5-vertex nonfully connected microwave network. We have shown that the spectrum of a simple microwave network also gives, through the Euler characteristic, an insight into the number $\beta=1-\chi$ of independent cycles. Since, according to Kuratowski's theorem [58], the planar systems are characterized by $\beta \leq 3$, we used the number $\beta$ of independent cycles as an independent revealer of planar and non-planar graphs.

\section{Acknowledgments}

This work was supported in part by the National Science Centre, Poland (grant No. 2016/23/B/ST2/03979), the Swedish Research Council (grant 2020-03780) and the Center for Interdisciplinary Research $(\mathrm{ZiF})$ in Bielefeld within the framework of the cooperation group on Discrete and Continuous Models in the Theory of Networks.

\section{References}

[1] L. Euler, Comment. Acad. Sci. U. Petrop 8, 128 (1736).

[2] L. Pauling, J. Chem. Phys. 4, 673 (1936).

[3] P. Exner, P. Šeba, P. Št'ovíček, J. Phys. A 21, 4009 (1988).

[4] T. Kottos, U. Smilansky, Phys. Rev. Lett. 79, 4794 (1997).

[5] R. Blümel, Yu. Dabaghian, R.V. Jensen, Phys. Rev. Lett. 88, 044101 (2002).

[6] G. Berkolaiko, P. Kuchment, Introduction to Quantum Graphs, Mathematical Surveys and Monographs 186, 2013, p. 270.

[7] Z. Pluhař, H.A. Weidenmüller, Phys. Rev. Lett. 112, 144102 (2014).

[8] D. Kowal, U. Sivan, O. Entin-Wohlman, Y. Imry, Phys. Rev. B 42, 9009 (1990).

[9] Y. Imry, Introduction to Mesoscopic Systems, Oxford 1996.

[10] J.A. Sanchez-Gil, V. Freilikher, I. Yurkevich, A.A. Maradudin, Phys. Rev. Lett. 80, 948 (1998).

[11] R. Mittra, S.W. Lee, Analytical Techniques in the Theory of Guided Waves, Macmillan, New York 1971.

[12] M. Ławniczak, P. Kurasov, S. Bauch, M. Białous, V. Yunko, L. Sirko, Phys. Rev. E 101, 052320 (2020).
[13] O. Hul, S. Bauch, P. Pakoński, N. Savytskyy, K. Życzkowski, L. Sirko, Phys. Rev. E 69, 056205 (2004).

[14] M. Ławniczak, O. Hul, S. Bauch, P. Šeba, L. Sirko, Phys. Rev. E 77, 056210 (2008).

[15] O. Hul, M. Ławniczak, S. Bauch, A. Sawicki, M. Kuś, L. Sirko, Phys. Rev. Lett 109, 040402 (2012).

[16] M. Ławniczak, S. Bauch, L. Sirko, in: Handbook of Applications of Chaos Theory Eds. Christos Skiadas, Charilaos Skiadas, CRC Press, Boca Raton (USA) 2016, p. 559 .

[17] B. Dietz, V. Yunko, M. Białous, S. Bauch, M. Ławniczak, L. Sirko, Phys. Rev. E 95. 052202 (2017).

[18] M. Ławniczak, L. Sirko, Sci. Rep. 9, 5630 (2019).

[19] M. Ławniczak, J. Lipovský, L. Sirko, Phys. Rev. Lett. 122, 140503 (2019).

[20] A. Rehemanjiang, M. Allgaier, C.H. Joyner, S. Müller, M. Sieber, U. Kuhl, H.-J. Stöckmann, Phys. Rev. Lett. 117, 064101 (2016).

[21] J. Lu, J. Che, X. Zhang, B. Dietz, Phys Rev. E 102, 022309 (2020).

[22] M. Ławniczak, S. Bauch, O. Hul, L. Sirko, Phys. Rev. E 81, 046204 (2010).

[23] M. Allgaier, S. Gehler, S. Barkhofen, H.-J. Stöckmann, U. Kuhl, Phys. Rev. E 89, 022925 (2014).

[24] M. Białous, V. Yunko, S. Bauch, M. Ławniczak, B. Dietz, L. Sirko, Phys. Rev. Lett. 117, 144101 (2016).

[25] M. Ławniczak, M. Białous, V. Yunko, S. Bauch, B. Dietz, L. Sirko, Acta Phys. Pol. A 132, 1672 (2017).

[26] H.J. Stöckmann, J. Stein, Phys. Rev. Lett. 64, 2215 (1990).

[27] S. Sridhar, A. Kudrolli, Phys. Rev. Lett. 72, 2175 (1994).

[28] L. Sirko, P.M. Koch, R. Blümel, Phys. Rev. Lett. 78, 2940 (1997).

[29] Y. Hlushchuk, A. Kohler, Sz. Bauch, L. Sirko, R. Blümel, M. Barth, H.-J. Stöckmann, Phys. Rev. E 61, 366 (2000).

[30] Y. Hlushchuk, A. Błędowski, N. Savytskyy, L. Sirko, Phys. Scr. 64, 192 (2001).

[31] R. Blümel, P.M. Koch, L. Sirko, Found. Phys. 31, 269 (2001).

[32] A. Dhar, D.M. Rao, U. Shankar, S. Sridhar, Phys. Rev. E 68, 026208 (2003).

[33] S. Hemmady, X. Zheng, E. Ott, T.M. Antonsen, S.M. Anlage, Phys. Rev. Lett. 94, 014102 (2005). 
[34] O. Hul, O. Tymoshchuk, S. Bauch, P.M. Koch, L. Sirko, J. Phys. A 38, 10489 (2005).

[35] S. Hemmady, X. Zheng, J. Hart, T.M. Antonsen, Jr., E. Ott, S.M. Anlage, Phys. Rev. E 74, 036213 (2006).

[36] B. Dietz, A. Richter, Chaos 25, 097601 (2015).

[37] M. Białous, B. Dietz, L. Sirko, Phys. Rev. E 100, 012210 (2019).

[38] B. Dietz, T. Klaus, M. Miski-Oglu, A. Richter, M. Wunderle, Phys. Rev. Lett. 123, 174101 (2019).

[39] R. Blümel, A. Buchleitner, R. Graham, L. Sirko, U. Smilansky, H. Walther, Phys. Rev. A 44, 4521 (1991).

[40] R.V. Jensen, S.M. Susskind, M.M. Sanders, Phys. Rep. 201, 1 (1991).

[41] M. Bellermann, T. Bergemann, A. Haffmanns, P.M. Koch, L. Sirko, Phys. Rev. A 46, 5836 (1992).

[42] L. Sirko, S. Yoakum, A. Haffmans, P.M. Koch, Phys. Rev. A 47, R782 (1993).

[43] A. Buchleitner, D. Delande, Phys. Rev. Lett. 71, 3633 (1993).

[44] L. Sirko, M.R.W. Bellermann, A. Haffmans, P.M. Koch, D. Richards, Phys. Rev. Lett. 71, 2895 (1993).

[45] J.E. Bayfield, S.-Y. Luie, L.C. Perotti, M.P. Skrzypkowski, Physica D Nonlin. Phenom. 83, 46 (1995).
[46] L. Sirko, P.M. Koch, Appl. Phys. B 60, S195 (1995).

[47] L. Sirko, A. Haffmans, M.R.W. Bellermann, P.M. Koch, Europhys. Lett. 33, 181 (1996).

[48] J.E. Bayfield, L.A. Pinnaduwage, J. Phys. $B$ 18, L49 (1999).

[49] L. Sirko, S.A. Zelazny, P.M. Koch, Phys. Rev. Lett. 87, 043002 (2001).

[50] L. Sirko, P.M. Koch, Phys. Rev. Lett. 89, 274101 (2002).

[51] A. Arakelyan, J. Nunkaew, T.F. Gallagher, Phys. Rev. A 94, 053416 (2016).

[52] P. Kurasov, Arkiv för Matematik 46, 95 (2008).

[53] P. Kurasov, J. Funct. Anal. 254, 934 (2008).

[54] B. Gutkin, U. Smilansky, J. Phys. A 34, 6061 (2001).

[55] P. Kurasov, M. Nowaczyk, J. Phys. A 38, 4901 (2005).

[56] J. von Below, Can one hear the shape of a network? Partial differential equations on multistructures, Luminy, 1999; Lecture Notes in Pure and Appl. Math., Vol. 219, Dekker, 2001, p. 19.

[57] M. Kac, Am. Math. Monthly 73, 1 (1966).

[58] K. Kuratowski, Fund. Math. 15, 271 (1930) (in French). 